\title{
Atypical Brain Calcifications Causing Seizure-Imaging Appearances
}

ABSTRACT

Introduction: Brain calcification causing seizure disorder is a common clinical condition. Calcification may be single or multiple. Seizure cases with calcifications reported in our institute are analysed in this article. There is diagnostic challenge if calcifications are multiple or in atypical locations. The Imaging appearances of calcification in $\mathrm{CT}, \mathrm{MRI}$ help a lot to arrive at definitive diagnosis.

Aim: To analyse the imaging appearances of brain calcifications causing seizure and to find out the commonest etiology and age group most commonal affected.

Materials and Methods: This is a retrospective analytical study of six months period from June 2015 to November 2015. The study was done in the Radiodiagnosis Department of Tirunelveli Medical College Hospital. In our institute, 3600 cases of CT brain have been taken in above period for various conditions. In which seizure patients with pathological calcifications who also underwent MRI brain were selected for the study i.e. sample selection. Total 78 patients were selected for analysis. The findings were analyzed and hereby are presented.

Results: Most of pathological calcifications belong to age group 21-40. Tuberculomas and tumors account for about $50 \%$ of cases. Both genders are equally affected. In MRI, T2*GRE sequence plays major role in identifying areas of calcification. Rare cases like unilateral hemispheric multiple cavernous angiomas, hypoparathyroidism and hemophilia sequlae are noted in this study.

Conclusion: The location of calcifications, extent, perilesional changes in the scans help to narrow down the differential diagnosis. MRI confirms the cavernous malformations. CT-scan is helpful for detecting calcifications. MRI is useful for diagnosing pericalcific parenchymal changes. Metabolic disorders should be ruled out if there is extensive bilateral cerebral and cerebellar calcification. Clinical history is very important for imaging interpretation as evident by the hemophiliac case.

\section{Keywords: Cavernoma, Hemophilia, Hypoparathyroidism, Magnetic resonance, Tomography}

\section{INTRODUCTION}

Seizure can occur due to various etiologies. Pathological calcifications contribute in significant number of cases. CT scan is highly sensitive for detecting calcifications and their location. MRI helps in detecting parenchymal changes associated with calcifications. Both modalities complement each other and help the radiologist to arrive at possible diagnoses. Intracranial calcifications can be classified mainly into 6 groups based on their etiopathogenesis: age- related and physiologic, congenital, infectious, endocrine and metabolic, vascular, and neoplastic. Intra cranial calcifications are common radiological findings. Knowledge regarding physiological calcifications is must to avoid misinterpretation. Imaging findings when combined with clinical findings in cases of calcifications help to narrow down the differential diagnosis, to identify the disease stage and in follow-up of the disease.

\section{MATERIALS AND METHODS}

This is a retrospective analytical study of six months period from June 2015 to November 2015. The study was done in the Radiodiagnosis Department of Tirunelveli Medical College Hospital, Tamil Nadu, India. In our institute, 3600 cases of CT brain have been taken in above period for various conditions.

Out of all 78 patients with pathological calcifications who also underwent MRI brain were selected for the study.

\section{Inclusion Criteria}

Patients with pathological calcifications in CT-scan upto the age of 80 years.

\section{Exclusion Criteria}

Patients who have not taken MRI, post- operative patients on follow-up, patients with history of head injury. Those 
findings were analyzed and hereby are presented. CT-scan was done with Toshiba single slice helical CT scanner. MRI was done with 1.5 tesla Siemens MRI scanner.

\section{RESULTS}

Radiology play a major role in detecting calcifications. In this study the selected patients have undergone both CT, MRI. The results are analyzed as follows. It is seen that most of pathological calcifications detected in the age group 21-40 years (44.86\%) out of all the cases [Table/Fig-1].

It is noted that tuberculomas and tumors account for about $50 \%$ of cases [Table/Fig-2]. It is seen that both genders are equally affected with pathological calcifications [Table/Fig-3]. We can see that T1, T2 W sequences pick up areas of calcifications in 38 patients (48.7\%) out of 78 cases [Table/Fig-4]. Rare cases like unilateral hemispheric multiple cavernous angiomas, hypoparathyroidism and hemophilia sequelae are noted in this study.

35 years old female was admitted with history of seizures in our hospital. Plain CT shows multiple intraparenchymal calcifications of varying sizes in right cerebral hemisphere [Table/Fig-5]. Also there is minimal right hemispheric atrophy. $\mathrm{MRI}$ shows multiple hyperintense lesions of varying sizes in

\begin{tabular}{|c|c|c|c|}
\hline S.No & Age group & Number of cases & Percentage (\%) \\
\hline 1 & $0-10$ & 12 & 15.38 \\
\hline 2 & $11-20$ & 10 & 12.82 \\
\hline 3 & $21-30$ & 19 & 24.35 \\
\hline 4 & $31-40$ & 16 & 20.51 \\
\hline 5 & $41-50$ & 7 & 8.97 \\
\hline 6 & $51-60$ & 5 & 6.41 \\
\hline 7 & $61-70$ & 5 & 6.41 \\
\hline 8 & $71-80$ & 4 & 5.12 \\
\hline \multicolumn{4}{|r|}{ Total } \\
[Table/Fig-1]: Age wise distribution. & 100 \\
\hline
\end{tabular}

\begin{tabular}{|c|l|c|}
\hline Sr no & Conditions & Number of cases \\
\hline 1 & HIE sequelae & 4 \\
\hline 2 & Tuberculoma & 21 \\
\hline 3 & Neurocysticercosis & 13 \\
\hline 4 & Cavernous malformation & 6 \\
\hline 5 & Aneurysm & 6 \\
\hline 6 & Tumors & 20 \\
\hline 7 & Sturge Weber Syndrome & 2 \\
\hline 8 & Tuberous sclerosis & 4 \\
\hline 9 & Hemophilia & 1 \\
\hline 10 & Hypoparathyroidism & 1 \\
\hline & Total & 78 \\
\hline
\end{tabular}

[Table/Fig-2]: Case wise distribution of pathological calcifications.

\begin{tabular}{|c|l|c|c|}
\hline S.no & Conditions & $\begin{array}{c}\text { Number of } \\
\text { male cases }\end{array}$ & $\begin{array}{c}\text { Number of } \\
\text { female cases }\end{array}$ \\
\hline 1 & HIE sequelae & 3 & 1 \\
\hline 2 & Tuberculoma & 8 & 13 \\
\hline 3 & Neurocysticercosis & 6 & 7 \\
\hline 4 & Cavernous malformation & 2 & 4 \\
\hline 5 & Aneurysm & 4 & 2 \\
\hline 6 & Tumors & 12 & 8 \\
\hline 7 & Sturge Weber Syndrome & 1 & 1 \\
\hline 8 & Tuberous sclerosis & 2 & 2 \\
\hline 9 & Hemophilia & 1 & - \\
\hline 10 & Hypoparathyroidism & 1 & - \\
\hline & Total & 40 & 38 \\
\hline [Table/Fig-3]: Gender wise distribution. & \\
\hline
\end{tabular}

\begin{tabular}{|c|l|c|c|}
\hline $\begin{array}{c}\text { Sr } \\
\text { no }\end{array}$ & Conditions & $\begin{array}{c}\text { Number of } \\
\text { cases detected } \\
\text { by TI, T2W seq }\end{array}$ & $\begin{array}{c}\text { Number of } \\
\text { cases Detected } \\
\text { by T1,T2 and } \\
\text { GRE seq }\end{array}$ \\
\hline 1 & HIE sequelae & 2 & 4 \\
\hline 2 & Tuberculoma & 9 & 21 \\
\hline 3 & Neurocysticercosis & 7 & 13 \\
\hline 4 & Cavernous malformation & 4 & 6 \\
\hline 5 & Aneurysm & 3 & 6 \\
\hline 6 & Tumors & 9 & 20 \\
\hline 7 & Sturge Weber Syndrome & 1 & 2 \\
\hline 8 & Tuberous sclerosis & 2 & 4 \\
\hline 9 & Hemophilia & 0 & 1 \\
\hline 10 & Hypoparathyroidism & 1 & 1 \\
\hline & Total & 38 & 78 \\
\hline & [Table/Fig-4]: Detectability of calcifications using MRI sequences. \\
\hline
\end{tabular}

T1W sequence. FLAIR MRI shows popcorn appearance with hemosiderin rim in most of the lesions without mass effect [Table/Fig-6]. MR Angiogram shows no significant feeding arteries [Table/Fig-7]. On gandolinium administration, there is no significant enhancement of the lesions. On analyzing above findings, the diagnosis arrived was multiple cavernous angiomas in the right cerebral hemisphere with minimal atrophic changes. Usually the cavernomas if multiple affect both hemispheres. This case is presented for its rarity of single hemispheric involvement.

Another 22 years old male was admitted in our hospital with seizure. Plain CT shows extensive brain calcifications involving deep grey matter like basal ganglia, thalamus and caudate nucleus [Table/Fig-8]. There is also subcortical white matter calcification. Cerebellum shows dense dentate nucleus calcifications bilaterally [Table/Fig-9]. MRI shows hyperintense areas in the basal ganglia in T1W sequence [Table/ Fig-10]. FLAIR shows hyperintensity in caudate and basal 

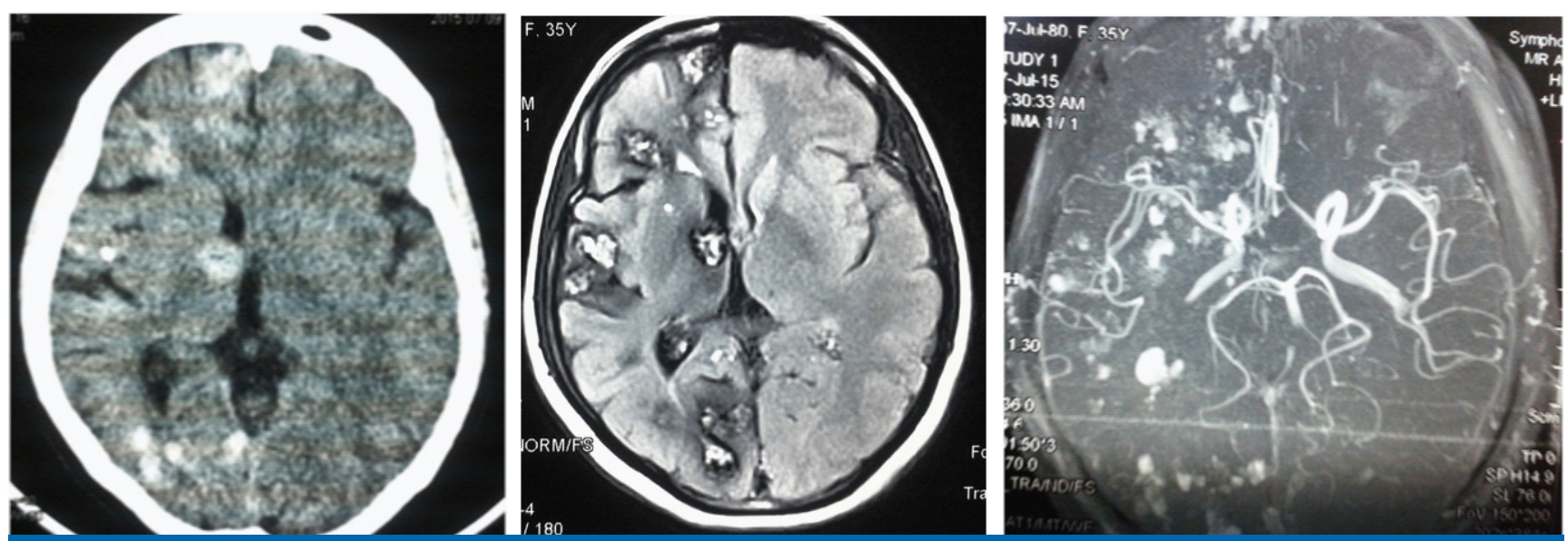

[Table/Fig-5]: Plain CT- multiple calcifications of varying sizes in right cerebral hemisphere. [Table/Fig-6]: FLAIR MRI-popcorn appearance with hemosiderin rim. [Table/Fig-7]: MR Angiogram-no significant feeding arteries.
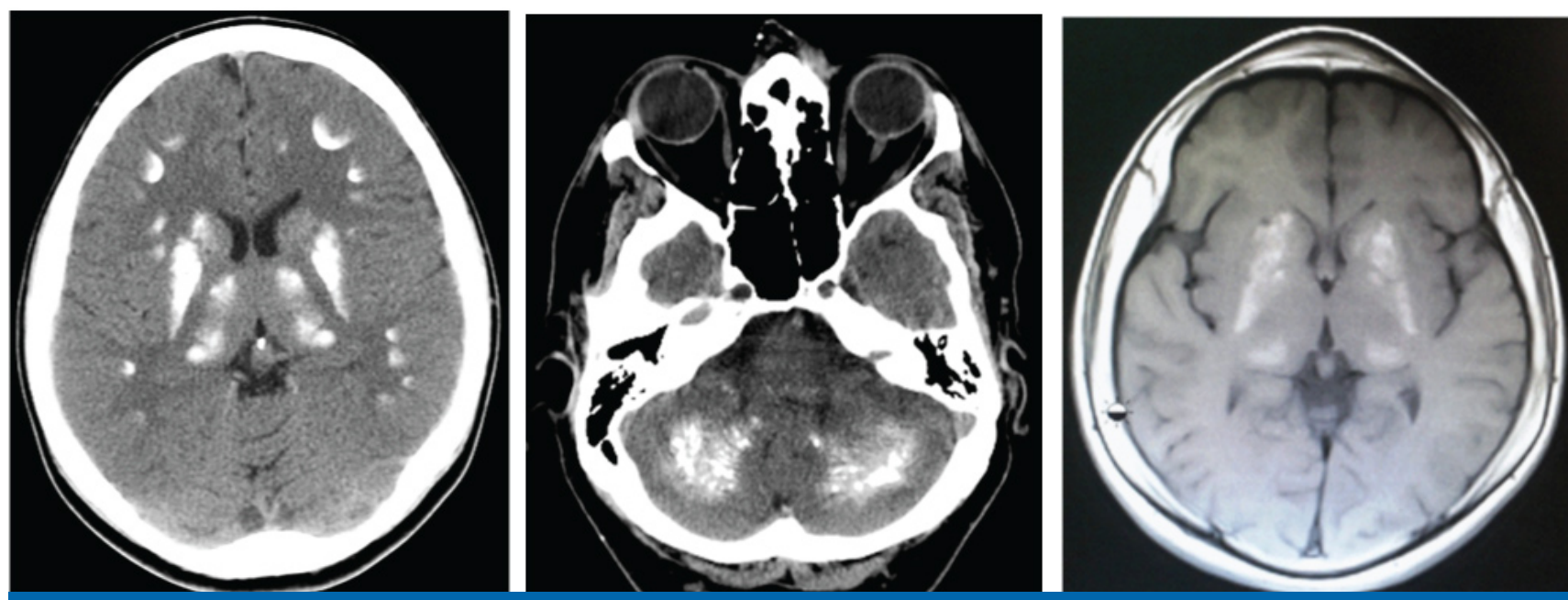

[Table/Fig-8]: Plain CT-bilateral calcifications involving basal ganglia, thalamus and caudate nucleus.

[Table/Fig-9]: Plain CT-bilateral dentate nuclei calcifications.

[Table/Fig-10]: T1W MRI-hyperintense areas in the basal ganglia, thalami.
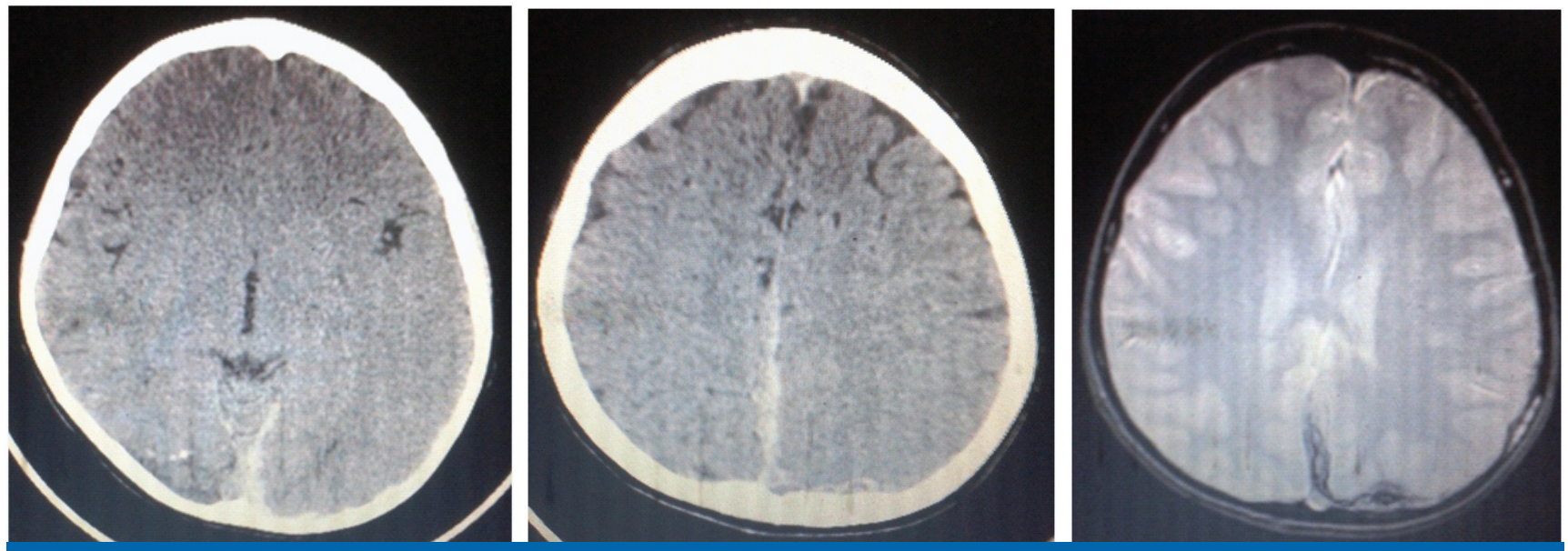

[Table/Fig-11]: Plain CT-right occipital lobe atrophy with tiny calcifications.

[Table/Fig-12]: Plain CT-dural calcification.

[Table/Fig-13]: GRE MRI-blooming of dural space. 
ganglia region. On interpreting above findings, radiological differential diagnoses are Fahr disease, metabolic disorders especially parathyroid disorders. X-ray hands of the patient shows osteopenia. ECG shows features of hypocalcemia. Paratharmone level was low in this patient. The diagnosis in this patient is hypoparathyroidism. This case is presented for highlighting the importance of ruling out metabolic conditions before arriving diagnosis of Fahr disease. Another case of seizure, 8 years old male boy was admitted in our hospital. CT shows right occipital lobe atrophy with tiny calcifications [Table/Fig-11]. CT also shows dural calcifications-HU 110-[Table/Fig-12]. MRI shows right occipital lobe atrophy. GRE sequences shows blooming of dura [Table/Fig-13]. Radiological diagnosis is old infarct in right occipital lobe with tiny calcifications and dural calcification.

The patient is known case of hemophilia. So the brain changes are sequelae of hemophilia bleed in dural space and in right occipital lobe. Seizure in this case may be due to calcifications or due to post gliotic scarring. This case highlights importance of clinical history.

\section{DISCUSSION}

Based on etiopathogenesis, there are six groups of intracranial calcifications [1]. They are as follow. Physiologic and age related causes- Pineal gland, habenula, sagittal sinus, choroid plexus, falx cerebri, dura mater, petroclinoid ligament and tentorium cerebelli. Infectious causes include granulomas, encephalitis, TORCH infections. Cavernous malformation, primary atherosclerosis, arteriovenous malformation, aneurysms, dystrophic in chronic infarction and chronic vasculitis are vascular causes [1]. Endocrine and metabolic etiologies are Fahr disease, hypoparathyroidism, hypothyroidism, post-thyroidectomy, hyperparathyroidism and pseudo-hypoparathyroidism. Neoplastic etiologies are oligodendroglioma, craniopharyngioma, germ cell neoplasms, calcified metastases, meningioma, choroid plexus papilloma, neurocytoma, dermoid, epidermoid, primitive neuroectodermal tumor (PNET), ependymoma, medulloblastoma, low grade astrocytoma. Congenital causes include Sturge-Weber syndrome, tuberous sclerosis, neurofibromatosis. Cavernomas or cavernous malformations are one of the vascular malformations of brain.

Solitary lesions are common. More than one occurs in one third of patients [2]. Although, enhancement is possible. The lesions generally do not enhance [3]. MRI is the modality of choice of detection. A characteristic "popcorn" or "berry" appearance with a rim of signal loss due to haemosiderin occurs in MRI. Prominent blooming on susceptibility weighted sequences also occur. In patients with familial or multiple cavernous angiomas GRE T2* sequences are very important in identifying the number of lesions. These lesions are now known as slow flow venous malformations according to newer nomenclature (ISSVA classification of vascular anomalies). The availability of MRI has increased the detection of cavernous malformations of the brain greatly [4]. Hypoparathyroidism causes multiple brain calcifications. Most commonly basal ganglia but also subcortical white matter, corona radiata and thalamus are affected [5]. The true mechanism behind the calcification in hypoparathyroidism is not completely understood [6]. Calcium deposits extent depends on duration and the stage of disease [7]. Other diagnoses that should be ruled out are Fahr disease, hyperparathyroidism, trisomy 21 or 5, Cockayne syndrome, radiation therapy and intrathecal chemotherapy. Hemophilia is a clinical condition with bleeding manifestations. Haemophilia $A$ is the cause of $80 \%$ of cases resulting from deficiency of coagulation Factor VIII. Haemorrhage following minor trauma or spontaneous haemorrhage occurs in $70 \%$ of patients of hemophilia $A$ [8]. Intracranial hemorrhage is an important complication for hemophiliac children [9]. One of the most common causes of death in hemophiliacs is Intracranial hemorrhage [10]. Hemophiliac arthropathy is the commonest presentation. Widening of intercondylar notch, squaring of the patella, joint space narrowing, osteoporosis, accelerated degenerative changes are the classical X-ray features of hemophilic knee. Intracranial bleeding occurs in subdural space, subarachnoid space, ventricles, cerebrum or cerebellum in hemophiliacs. Sometimes bleeding occurs in more than one site.

\section{LIMITATIONS OF THE STUDY}

1. The patients who have taken both CT and MRI only are analysed in this study.

2. SWI (susceptibility weighted imaging) in MRI is not done.

The limitations may be overcome by reducing cost of MRI imaging and addition of recent techniques i.e. SWI in MRI in imaging of seizure patients.

\section{CONCLUSION}

From this study, it is noted that most of pathological calcifications belong to age group 21-40. Tuberculomas and tumors account for about $50 \%$ of cases. Both genders are equally affected. In MRI, T2*GRE sequence plays major role in identifying areas of calcification. The location of calcifications, extent, perilesional changes in the scans help to narrow down the differential diagnosis. MRI confirms the cavernous malformations. CT scan is helpful for detecting calcifications. MRI is useful for diagnosing pericalcific parenchymal changes. Metabolic disorders should be ruled out if there is extensive bilateral cerebral and cerebellar calcification. Clinical history is very important for imaging interpretation as evident by above hemophiliac case. 


\section{REFERENCES}

[1] Kıroglu Y, Çallı C, Karabulut N, Öncel C. Intracranial calcifications on CT. Diagn Interv Radiol. 2010; 16:263-69.

[2] Blitstein MK, Tung GA. MRI of cerebral micro hemorrhages. AJR Am J Roentgenol.2007;189(3):720-25.doi:10.2214/AJR. 07.2249 .

[3] Pinker K, Stavrou I, Knosp E et al. Are cerebral cavernomas truly non-enhancing lesions and thereby distinguishable from arteriovenous malformations? MRI findings and histopathological correlation. Magn Reson Imaging. 2006; 24 (5): 631-37. doi:10.1016/j.mri.2005.10.037.

[4] Stacey RJ, Findlay GFG, Foy PM, Jeffreys RV. Cavernomas in the central nervous system and the relevance of multiple intracranial lesions in the familial form of this disease. $J$ Neurol Neurosurg Psychiatry. 1999;66:117. doi:10.1136/jnnp.66.1.117.

\section{AUTHOR(S):}

1. Dr. Nellaiappan Chelliah

\section{PARTICULARS OF CONTRIBUTORS:}

1. Associate Professor, Department of Radiodiagnosis, Tirunelveli Medical College Hospital, Tamilnadu, India.
[5] Adam A, Dixon AK, Gillard JH et-al. Grainger \& Allison's Diagnostic Radiology: Expert Consult: Online and Print. Churchill Livingstone. ISBN:B00K8G3JYK.

[6] Fujita T. Mechanism of intracerebral calcification in hypoparathyroidism. Clin Calcium. 2004; 14(6):55-57.

[7] Mejdoubi M. Extensive brain calcification in idiopathic hypoparathyroidism. J Neurol Neurosurg Psychiatry. 2006; 77(12):1328.

[8] National Heart, Lung, and Blood Institute-NHLBI Diseases and Conditions Index-. Hemophilia. Available at http://www.nhlbi. nih.gov/health/dci/Diseases/hemophilia/hemophilia_all.html.

[9] Nagel K, Pai MK, Paes BA, Chan AK. Diagnosis and treatment of intracranial hemorrhage in children with hemophilia. Blood Coagul Fibrinolysis. 2013;24(1):23-27. doi: 10.1097/MBC.

[10] Yeung JTH, Lee CM, Lo AXN, Ma JKF and Fong JCY. Common and uncommon imaging manifestations of hemophilia, Appl Radiol. February 01, 2013.

\section{NAME, ADDRESS, E-MAIL ID OF THE CORRESPONDING AUTHOR:}

Dr. Nellaiappan Chelliah,

Associate Professor, Department of Radiodiagnosis,

Tirunelveli Medical College Hospital, Tamil Nadu-627011, India.

E-mail: cnellaiappan@yahoo.co.in

FINANCIAL OR OTHER COMPETING INTERESTS:

None.

Date of Online Ahead of Print: Mar 04, 2016 Date of Publishing: Apr 01, 2016 